\title{
Prevalence of Salmonella spp. in red-footed tortoises (Chelonoidis carbonaria) from Grenada, West Indies
}

Ravindra Nath Sharma ${ }^{1}$, Vanessa Matthew-Belmar ${ }^{1}$, Roxane Nicholas-Thomas ${ }^{1}$, Gitanjali Arya ${ }^{2}$, Bob Holtslander ${ }^{2}$, Harry Hariharan ${ }^{1}$ and Victor A. Amadi ${ }^{1}$

1. Department of Pathobiology, School of Veterinary Medicine, St. George's University, Grenada, West Indies; 2. Office International des Epizooties Salmonella Reference Laboratory, Public Health Agency of Canada, National Microbiology Laboratory at Guelph, Guelph, Ontario, Canada.

Corresponding author: Victor A. Amadi, e-mail: vamadi@sgu.edu

Co-authors: RNS: rsharma@sgu.edu, VM: vmatthew@sgu.edu, RN: rnichola@sgu.edu, GA: gitanjali.arya@canada.ca, BH: bob.holtslander@canada.ca, HH: hhariharan@sgu.edu

Received: 03-07-2019, Accepted: 26-12-2019, Published online: 22-01-2020

doi: www.doi.org/10.14202/IJOH.2020.12-16 How to cite this article: Sharma RN, Matthew-Belmar V, NicholasThomas R, Arya G, Holtslander B, Hariharan H, Amadi VA (2020) Prevalence of Salmonella spp. in red-footed tortoises (Chelonoidis carbonaria) from Grenada, West Indies, Int J One Health, 6(1): 12-16.

\begin{abstract}
Background and Aim: There is currently no published information on the prevalence of Salmonella spp. in pet red-footed tortoises in Grenada. Monitoring is essential to understand the dynamics of the epidemiology of Salmonella. This study aimed to estimate the prevalence of Salmonella spp. from pet red-footed tortoises from Grenada and to test for antimicrobial drug resistance of the isolates.
\end{abstract}

Materials and Methods: Established bacterial culture, serotyping, and antimicrobial susceptibility methods were used. Fecal samples were obtained from 114 tortoises from five parishes of Grenada, and cultured for Salmonella using enrichment and selective culture techniques. The serotyping of isolated Salmonella was performed at the Office International des Epizooties Salmonella Reference Laboratory, Guelph, Ontario, Canada. The isolates were tested for antimicrobial susceptibility in the Microbiology Laboratory of the School of Veterinary Medicine, St. George's University, Grenada.

Results: Fifteen tortoises (13.2\%) out of 114 were positive for Salmonella spp. Five serovars were identified: Salmonella Javiana (6.3\%), Salmonella Rubislaw (6.3\%), Salmonella Saintpaul (18.75\%), Salmonella Glostrup (6.3\%), and S. I: Rough.O;e,h:1,2 (62.5\%). All isolates were sensitive to the 12 antimicrobial drugs.

Conclusion: Three serovars ( $S$. Javiana, $S$. Saintpaul, and $S$. Rubislaw) are known pathogens causing disease in humans. Since tortoises included in the study were pets, the tortoises may be a possible source of transmission of Salmonella to their owners. The tortoise owners should be educated on the importance of hygienic raising of their pets. This is the first report of isolation of Salmonella spp. from pet red-footed tortoises in Grenada.

Keywords: antimicrobial susceptibility, Grenada, red-footed tortoises, Salmonella spp.

\section{Introduction}

Foodborne diseases in mammals and birds are a serious problem worldwide. Ingestion of contaminated food and water with zoonotic pathogens including Salmonella spp. can cause serious diseases in humans and animals.

Recently, Maria et al. [1] while reviewing foodborne diseases in the Caribbean reported Salmonella spp. as the main cause of the disease in Trinidad and Tobago [2], Dominica [3], St. Lucia [4], Grenada [5], and Barbados [6]. Reptiles including various species of tortoises have been shown to carry zoonotic pathogens including Salmonella spp. from different parts of the world including pet turtles in Korea [7], Russian tortoises in Spain [8], Russian tortoises in Poland [9],

Copyright: Sharma, et al. This article is an open access article distributed under the terms of the Creative Commons Attribution 4.0 International License (http://creativecommons.org/licenses/ by/4.0/), which permits unrestricted use, distribution, and reproduction in any medium, provided you give appropriate credit to the original author(s) and the source, provide a link to the Creative Commons license, and indicate if changes were made. The Creative Commons Public Domain Dedication waiver (http:// creativecommons.org/ publicdomain/zero/1.0/) applies to the data made available in this article, unless otherwise stated. gopher tortoises in Georgia [10] and in Florida [11], desert tortoises in Arizona [12], European tortoises [13], red-foot tortoises in Brazil [14], captive reptiles from Croatia [15], and from reptiles in Taiwan [16].

In Grenada, Salmonella spp. has been reported from wild animal species, including cane toads [17], blue land crabs [18], small Indian mongoose [19], green iguana [20], and brown rats [21]. There is no published report on the isolation of Salmonella spp. from tortoises in Grenada.

This study was undertaken with two objectives: (1) To estimate the prevalence of Salmonella spp. and their serovars in red-footed tortoises, and (2) to determine the antimicrobial resistance of the Salmonella isolates.

\section{Materials and Methods}

\section{Ethical approval and informed consent}

The research project was approved by the Institutional Animal Care and Use Committee (IACUC) of St. George's University (IACUC 18009. $23^{\text {rd }}$ August 2018). Tortoise owners were randomly selected from five out of the six parishes of the Island of Grenada based on their availability and the acceptance of the owners to include their tortoise in this 
study. The research plan was explained to the owners in detail, and written consent was obtained from those who agreed to participate in the research before the collection of samples from the randomly selected tortoises.

\section{Study area}

Grenada is the southernmost country in the Caribbean sea with an area of $348.5 \mathrm{~km}^{2}$. The country with low hills, small trees, shrubs, and tropical climate is most suitable for the habitat of tortoises. The environmental temperature remains within a range of $20^{\circ} \mathrm{C}-30^{\circ} \mathrm{C}$, preferred climate for red-footed tortoises. The country is divided into six parishes; St. Patrick, St. Mark, St. Andrew, St. John, St. George, and St. David. The terrain throughout the country is similar.

\section{Collection of fecal samples}

A cross-sectional study was performed to meet the aim of the research on the population of pet tortoises in Grenada. Demographic information on the location of tortoises in different parishes, gender, and age were taken at the time of sample collection. Fecal samples from 114 tortoises were collected using fecal swabs in the Cary-Blair Transport Medium (Becton, Dickinson and Co., Sparks, MD, USA) during a period of 5 months, November 2018-March 2019. The fecal swabs were transported to the Microbiology Laboratory of School of Veterinary Medicine and cultured the same day. For the collection of fecal swabs, the tested tortoise was put on its back on a hard surface and cloaca was stimulated by gentle finger touch.

\section{Bacterial culture and serotyping}

Recommended culture method for the isolation of Salmonella by Gorski et al. [22] was used with slight modification, as described by Drake et al. [17] and Sylvester et al. [20]. Briefly, $0.1 \mathrm{ml}$ intestinal content was inoculated into $10 \mathrm{ml}$ of tryptic soy broth (TSB; Remel Lenexa, KS) a non-selective medium and incubated at $37^{\circ} \mathrm{C}$ for $24 \mathrm{~h}$. After incubation, an aliquot $(0.1 \mathrm{ml})$ of the TSB was inoculated into $10 \mathrm{ml}$ of Rappaport-Vassiliadis (RV) broth (Difco, BD, Sparks, MD) which is selective enrichment medium for Salmonella. The culture was incubated at $42^{\circ} \mathrm{C}$ for $48 \mathrm{~h}$. Subcultures of the RV broth culture were made on xylose lysine desoxycholate selective indicator agar (Difco) and incubated at $37^{\circ} \mathrm{C}$ for 18-24 h. To increase the chances of isolation of multiple serovars of Salmonella, up to five individual colonies with typical Salmonella morphology (red translucent colonies with black centers) were subcultured on tryptic soy agar (TSA) plates. Bacterial colonies from TSA plates were tested for agglutination using Salmonella O Antiserum Poly A-1 and Vi (Difco BD). All agglutination positive cultures were inoculated into API 20E (Analytical Profile Index, BioMerieux Inc. Durham, NC) strips and incubated at $37^{\circ} \mathrm{C}$ for $18-24 \mathrm{~h}$ for confirmation of Salmonella spp. Salmonella Typhimurium ATCC 14028 was used as a reference culture. Pure Salmonella cultures were stored in TSA slants and shipped in cold packed containers for serotyping to the World Organization for Animal Health (Office International des Epizooties; OIE) Salmonella Reference Laboratory of the Public Health Agency of Canada's National Microbiology Laboratory at Guelph Ontario, Canada, using established methods $[23,24]$. The serovars were named as per the antigenic formulae listed by Grimont [25].

\section{Antimicrobial susceptibility testing}

Antimicrobial susceptibility testing was performed as previously described [26] as recommended by the Clinical and Laboratory Standards Institute (CLSI 2015) [27]. The antibiotic disks used in this research were: Amoxicillin clavulanic acid (AmC) $30 \mu \mathrm{g}$, ampicillin (AM) $10 \mu \mathrm{g}$, chloramphenicol (C) $30 \mu \mathrm{g}$, ceftazidime (CAZ) $30 \mu \mathrm{g}$, cefotaxime (CTX) $30 \mu \mathrm{g}$, cephalothin (CF) $30 \mu \mathrm{g}$, ciprofloxacin (CIP) $5 \mu \mathrm{g}$, imipenem (IPM) $10 \mu \mathrm{g}$, gentamicin (GM) $10 \mu \mathrm{g}$, neomycin (NM) $30 \mu \mathrm{g}$, tetracycline (TE) $30 \mu \mathrm{g}$, and trimethoprim/sulfamethoxazole $25 \mu \mathrm{g}$. Antibiotics disks were obtained from BD, Franklin Lakes, NJ. The CLSI guidelines were used for the interpretation of the inhibition zone size for all antibiotics except for neomycin which as interpreted based on the guideline from manufacturer zone [26,27]. Escherichia coli ATCC 25922 was used as a quality control strain [26,28].

\section{Statistical analysis}

The data were analyzed by the statistical analysis: Fisher's exact test using graphical statistical software (http://www.graphpad.com/quickcales/contingency2).

\section{Results}

Salmonella spp. was isolated from 15 (13.2\%) out of 114 tortoises tested. Single serotype was isolated from 14 tortoises and one tortoise had two serotypes, making a total of 16 serotypes. In one tortoise, mixed infection with Salmonella Saintpaul and S. I: Rough.O;e,h:1,2 was found. Salmonella serovars were Salmonella Javiana, Salmonella Rubislaw, S. Saintpaul, Salmonella Glostrup, and $S$. I: Rough.O;e,h:1,2. Results of Salmonella isolation from different parishes of Grenada are included in Tables-1 and 2.

All Salmonella serovars isolated in the present study were found susceptible to all 12 antimicrobial drugs.

\section{Discussion}

The present study revealed a $13.2 \%$ prevalence of Salmonella spp. in red-footed tortoises from Grenada. Five serovars $S$. Javiana, $S$. Glostrup, $S$. Rubislaw, $S$. Saintpaul, and $S$. I: Rough.O;e,h:1,2 were identified from the isolates. There is a paucity of Salmonella research on red-footed tortoises. However, our findings were comparable to Oberdan et al. [14] who reported a $12.3 \%$ prevalence of Salmonella spp. in red-footed tortoise from Brazil. The prevalence of Salmonella spp. in gopher tortoises (Gopherus polyphemus) has been reported in South Georgia 2.6\% [10] and Florida 
Table-1: Isolation of Salmonella Spp. from red-footed tortoises in Grenada.

\begin{tabular}{|c|c|c|c|c|c|c|c|c|}
\hline Parish & Male tested & Positive & Female tested & Positive & Unknown sex tested & Positive & Total & Positive \\
\hline St. Andrew & 8 & 0 & 8 & 0 & 6 & 0 & 22 & 0 \\
\hline St. George & 20 & 1 & 24 & 1 & 8 & 0 & 52 & $2(3.9 \%)$ \\
\hline St. David & 2 & 1 & 8 & 1 & 0 & 0 & 10 & $2(20 \%)$ \\
\hline St. John & 6 & 0 & 7 & 0 & 0 & 0 & 13 & 0 \\
\hline St. Patrick & 14 & 9 & 2 & 1 & 1 & 1 & 17 & $11(64.7 \%)$ \\
\hline Total & 50 & $11(22 \%)$ & 49 & $3(6.1 \%)$ & 15 & $1(6.7 \%)$ & 114 & $15(13.2 \%)$ \\
\hline
\end{tabular}

Table-2: Salmonella serovars from red-footed tortoises in Grenada.

\begin{tabular}{lcl}
\hline Serovar & Number (\%)* & Parishes \\
\hline Salmonella Javiana & $1(6.3)$ & St. Patrick \\
Salmonella Glostrup & $1(6.3)$ & St. George \\
Salmonella Rubislaw & $1(6.3)$ & St. George \\
Salmonella Saintpaul & $3(18.75)$ & St. David (1) \\
& & St. Patrick (2) \\
S. I: Rough.0;e,h:1,2 & $10(62.5)$ & St. David (1) \\
& & St. Patrick (9) \\
\hline
\end{tabular}

*Salmonella Saintpaul and S. I: Rough.O;e,h:1,2 were isolated from one positive tortoise

$10 \%$ [11], and in Russian tortoises in Poland, 18.5\% [9]. The variation in the prevalence of Salmonella spp. among different breeds and places could be because of their different living environments [29].

In the present study, $S$. I: Rough.O;e,h:1,2(62.5\%) was the most common serovar isolated. Approximately $90 \%$ of $S$. I: Rough.O;e,h:1,2 were confined to one parish, St. Patrick. S. I: Rough.O;e,h:1,2 lack O antigen and are untypable. To know the pathogenicity and clinical significance of untypable isolates, they need to be further studied.

$S$. Saintpaul was isolated from $18.7 \%$ tortoises, in the present study. In Grenada, $S$. Saintpaul was isolated from blue land crab [18]. This serovar is known as the common cause of human salmonellosis in Europe, Australia, and the USA [30].

In the present investigation, $S$. Javiana serovar was isolated from one tortoise $(6.7 \%)$. S. Javiana has been reported as the most common cause of Salmonella outbreaks in humans [31]. S. Javiana has been associated with other wildlife species such as amphibians [32,33] and marsupial [34]. S. Javiana has been previously isolated from gopher tortoises [10], and red-footed tortoises [14]. In Grenada, $S$. Javiana has been reported in mongoose [19] and cane toads [17]. Ravindra et al. [21] reported $S$. Javiana as the most common serovar in brown rats from Grenada.

One tortoise was positive for $S$. Rubislaw $(6.7 \%)$ in the present investigation. This serovar has been reported from humans in Georgia [35] and in Florida [36]. Human cases have been reported as a result of exposure to reptile and amphibians [37]. In Grenada, $S$. Rubislaw has been isolated from wild species in cane toads [17] and mongoose [19].

A new serovar, $S$. Glostrup was isolated from one tortoise. Published literature is not available on its pathogenicity and the relationship to human cases.
Further study is needed to elucidate the characteristics of $S$. Glostrup serovar.

In the present study, the isolation of Salmonella spp. was significant $(P=0.02)$ higher in male tortoises (22\%) compared to females (6.1\%) (Table-1). Contrary to our findings, Lockhart et al. [10] and Charles et al. [11] reported no difference in the prevalence of Salmonella spp. infection in gopher tortoises in relation to sex. A further study involving a large population of red-footed tortoises is suggested to highlight the effect of gender on the prevalence of Salmonella spp. infection.

All isolates including $S$. I: Rough.O;e,h:1,2 were subjected to antibiotic sensitivity test in the study. All strains were sensitive to 12 antimicrobial drugs used in the test, including third-generation cephalosporins, and ciprofloxacin, the most commonly used drugs for human salmonellosis. Similar to our findings, Charles et al. [11] reported all Salmonella isolates from gopher tortoises from Florida susceptible to ten commonly used antimicrobial drugs. Chun-Yu et al. [16] found $S$. Typhimurium isolated from turtles in Taiwan, showing a wide range of resistance to ampicillin, chloramphenicol, gentamicin, streptomycin, trimethoprim-sulfamethoxazole, and tetracycline. Variable results for antimicrobial drugs for Salmonella spp. isolates in wildlife have been observed by the previous researchers [38]. Lack of exposure of wildlife to antimicrobial drugs may be the reason for the variability of the result in different studies. Long-term studies on the antimicrobial resistance in wildlife may elucidate this complex phenomenon.

\section{Conclusion}

In the present study, three pathogenic Salmonella serovars, namely, $S$. Javiana, $S$. Rubislaw, and $S$. Saintpaul were isolated from red-footed tortoises in Grenada. These serovars have previously been isolated from wildlife in Grenada. Since tortoises included in the present study were pets, Salmonella positive tortoises may be a possible source of infection to their owners. The tortoise owners should be educated on the importance of hygiene after raising and handling of their pets. This is the first report of isolation of Salmonella spp. from pet red-footed tortoises in Grenada.

\section{Authors' Contributions}

This research was carried out in collaboration between all authors. RNS designed the study, oversaw the research, and wrote the first draft of the 
manuscript. HH supervised the bacteriological work and finalized the manuscript. VAA organized the collection of samples and supervised the laboratory work, $\mathrm{RN}$ performed bacteriology of samples, $\mathrm{VM}$ helped in bacterial culture, performed antimicrobial sensitivity test and kept records, GA, and BH performed serotyping and identification of serovars. All authors revised, read and approved the final manuscript.

\section{Acknowledgments}

This study was funded by the St. George's University Small Research Grant Initiative (GSP $\backslash$ SRGI $\backslash 18011)$. The authors thank Dr. Alfred Chikweto Associate Professor, Pathology for help in the statistical analysis of data, and Ziebell Kim (OIE Salmonella Reference Laboratory, Canada) for help in the serotyping and identification of serovars.

\section{Competing Interests} interests.

The authors declare that they have no competing

\section{Publisher's Note}

Veterinary World (Publisher of International Journal of One Health) remains neutral with regard to jurisdictional claims in published institutional affiliation.

\section{References}

1. Maria, N.M.G., Andre, M.A. and Arve, L.W. (2016) An overview of food safety and bacterial Foodborne zoonosis in food-producing animals in the Caribbean region. Trop. Anim. Health Prod., 48(6): 1096-1108.

2. Lakhan, C., Badrie, N., Ramaubhag, A., Sundaraneedi, K. and Inder, L. (2013) Burden and impact of acute gastroenteritis and foodborne pathogens in Trinidad and Tobago. J. Health Popul. Nutr., 31(4): 30-42.

3. Ahmed, S., Ricketts, P., Bergeron, M., Jones, W. and Indar, L. (2013) Distribution, burden and impact of acute gastroenteritis in Dominica 2009-2010. J. Health Prod. Nutr., 31(4): 43-58.

4. Gabriel, O.O., Jaime, A., McKenzie, M., Auguste, A., Perez, E. and Indar, L. (2013) Estimating the burden of acute gastrointestinal illness: A pilot study of the prevalence and under-reporting in St Lucia Eastern Caribbean. $J$. Health Popul. Nutr., 31(4): 3-16.

5. Glasgow, L.M., Forde, M.S., Antoine, S.C., Perez, E. and Indar, L. (2013) Estimating the burden of acute gastroenteritis illness in Grenada. J. Health Popul. Nutr., 31(4): 17-29.

6. Kumar, A., Browne, C., Scotland, S., Krishnamurthy, K. and Nelsen, A.L. (2014) Selected enteropathogens and clinical course in children hospitalized with severe gastroenteritis in Barbados. Int. J. Health Sci. Quassin Univ., 8(4): 410-417.

7. Du-San, B., Gee-Wook, S., Mitchell, W. and Gang-Joon, H. (2016) Prevalence of Salmonella spp in pet turtles and their environment. Lab. Anim. Res., 32(3): 166-170.

8. Aneta, N., Grazyna, Z., Przemyslaw, Z. and Atanislaw, T. (2012) Russian tortoises (Agrionemys) as a potential reservoir for Salmonella spp. Res. Vet. Sci., 92(2): 187-190.

9. Nowakiewicz, A., Ziolkowska, G., Zieba, P., Stepniewska, K. and Tokarzewski, S. (2012) Russian tortoises (Agrionemys horsfieldi) as a potential reservoir for Salmonella spp. Res. Vet. Sci., 92(2): 187-190.

10. Lockhart, J.M., Lee, G., Turco, J. and Chamberlin, L. (2008) Salmonella from gopher tortoises (Gopherus polyphemus) in South Georgia. J. Wildl. Dis., 44(4): 88-991.

11. Charles, S.L.E., Lewbart, G.A., Aresco, M.J. and Peter, C. (2009) Detection of Salmonella in gopher tortoises (Gopherus polyphemus) during two relocation efforts in Florida. Chelonian Conserv. Biol., 8(2): 213-216.

12. Dickinson, V.M., Duck, T., Schwalbe, C.R., Jarchow, J.L. and Trueblood, M.H. (2001) Nasal and cloacal bacteria in free-ranging desert tortoises from the Western United States. J. Wildl. Dis., 37(2): 252-257.

13. Lecis, R., Paglietti, B., Rubino, S., Are, B.M., Muzzeddu, M., Berlinquer, F., Chessa, B., Pittau, M. and Alberti, A. (2011) Detection and characterization of Mycoplasma graeca, $T$. marginata. J. Wildl. Dis., 47(3): 717-724.

14. Oberdan, C.N., Eugenia, D.O., Sonia, S.L., Janis, C.H., Moacyr, M.N. and Carlos, RF. (2010) Isolmaneto E identificac cao De Salmonella sp. De jabutis Piranga (Chelonoidis Carbanaria) orindos do trafico De animals silvestres. Cienc. Anim. Bras., 11(1): 168-173.

15. Lukac, M., Pedersoen, K. and Prukner-Radovcic, E. (2015) Prevalence of Salmonella in captive reptiles from Croatia. J. Zoo. Wildl. Med., 46(2): 234-240.

16. Chun-Yu, C., Wan-Ching, C., Shih-Chiem, C., Yen-Hsueh, L., Kwong-Chung, T., Chien-Shun, C., YuanMan, H. and Chao-Chin, C. (2010) Prevalence and antimicrobial susceptibility of Salmonella isolates from reptiles in Taiwan. J. Vet. Diagn. Invest., 22(1): 44-50.

17. Drake, M., Amadi, V., Zieger, U., Johnson, R. and Hariharan, H. (2013) Prevalence of Salmonella spp. in cane toads (Bufo marinus) from Grenada., West Indies and their antimicrobial susceptibility. Zoonoses Public Health, 60(6): 437-441.

18. Peterson, R., Hariharan, H., Matthew, V., Chappel, S., Davies, R., Parker, S. and Sharma, R. (2013) Prevalence, serovars and antimicrobial susceptibility of Salmonella isolated from blue land crabs (Cardisoma guanhumi) in Grenada, West Indies. J. Food Prot., 76(7): 1270-1273.

19. Miller, S., Amadi, V., Stone, D., Johnson, R., Hariharan, H. and Zieger, U. (2014) Prevalence and antimicrobial susceptibility of Salmonella spp. in small Indian mongoose (Herpestes auropunctatus) in Grenada, West Indies. Comp Immunol Microb. Infect. Dis., 37(4): 205-210.

20. Sylvester, W., Amadi, V., Pinckney, R., Macpherson, C., McKibben, J., Bruhl-Day, R., Johnson, R. and Hariharan, H. (2014) Prevalence, serovars, and antimicrobial susceptibility of Salmonella spp. from wild and domestic green iguana (Iguana iguana) in Grenada, West Indies. Zoonoses Public Health, 61(6): 436-441.

21. Ravindra, S., Tiwari, K., Gitanjali, A., Victor, A., Darnell, B., Nicholas, M., Shua-Halm, T., Nicholas, T.R. and Hariharan, H. (2018) Salmonella serovars isolated from brown rats (Rattus norvegicus) from Grenada, West Indies: Prevalence and antimicrobial susceptibility. Microbiol. Res. J. Int., 25(1): 1-8.

22. Gorski, L., Craig, T.P., Anita, L., Michael, B.C., Michael, T.J., Andrew, G.G., Atwill, E.R. and Robert, E.M. (2011) Prevalence, distribution and diversity of Salmonella Enterica in a major production region of California. Appl. Environ. Microbiol., 77(8): 2734-2748.

23. Ewing, W.H. (1986) Edwards and Ewing's Identification of Enterobacteriaceae. $4^{\text {th }}$ ed. Elsevier Science Publishing Co., Inc., New York, USA.

24. Shipp, C.R. and Rowe, B. (1980) A mechanized microtechnique for Salmonella serotyping. J. Clin. Pathol., 33(6): 595-597.

25. Grimont, P.A.D. (2007) Antigenic formulas of the Salmonella serovars. In: WHO Collaborating Centre for Reference and Research on Salmonella. Institut Pasteur, Paris CEDEX, France.

26. Amadi, V.A., Hariharan, H., Arya, G., Matthew-Belmar, V., Nicholas-Thomas, R., Pinckney, R., Sharma, R. and Johnson, R. (2018) Serovars and antimicrobial resistance of non-typhoidal Salmonella isolated from non-diarrhoeic 
dogs in Grenada, West Indies. Vet. Med. Sci., 4(1): 26-34.

27. Clinical Laboratory Standards Institute. Performance Standards for Antimicrobial Disk and Dilution Susceptibility Test for Bacteria Isolated from Animals: Documents Vet01-S2. $3^{\text {rd }}$ ed. 2015. Second Informational Supplement. Clinical Laboratory Standards Institute, Wayne, PA, USA.

28. Eguale, T., Gebreyes, W.A., Asrat, D., Alemayhu, H., Gunn, J.S. and Engidawork, E. (2015) Non-typhoidal Salmonella serotypes, antimicrobial resistance and co-infection with parasites among patients with diarrhea and other gastrointestinal complaints in Addis Ababa, Ethiopia. BMC Infect Dis., 15: 497.

29. Nakadai, A., Kuroki, T., Kato, Y., Suzuki, R., Yamai, S., Yaginuma, C., Shiotani, R., Yamanouchi, A. and Hayashidani, H. (2005) Prevalence of Salmonella spp. in pet turtles in Japan. J. Vet. Med. Sci., 67(1): 97-101.

30. Beautlich, J., Rodriguez, I., Schroeter, A., Kashbohrer, A., Helmuth, R. and Guerra, B.A. (2010) Predominant multidrug-resistant Salmonella enterica serovar Saint Paul clonal line in German Turkey and related food products. Appl. Environ. Microbiol., 76(11): 3657-3667.

31. Mezal, E.H., Stefanova, R. and Khan, A.A. (2013) Isolation and molecular characterization of Salmonella Enterica serovar Javiana from food, environmental and clinical samples. Int. J. Food Microbiol., 164(1): 113-118.

32. Srikantiah, P., Lay, J.C., Hand, S., Crump, J.A., Campbell, J., Van Duyne, M.S., Bishop, R., Middender, R., Currier, M.,
Mead, P.S. and Molbak, K. (2004) Salmonella enterica serovar Javiana infections associated with amphibian contact, Mississippi 2001. Epidemiol. Infect., 132(2): 273-281.

33. Clarkson, L.S., Tobin-D’Angelo, M., Shuler, C., Hanna, S., Benson, J. and Voetsch, A.C. (2010) Sporadic Salmonella enterica serotype Javiana infections in Georgia and Tennessee: A hypothesis-generating study. Epidemiol. Infect., 138(3): 340-346.

34. Eveson, J.B. and Bradsaw, S.D. (1973) Salmonella Javiana infection in an infant associated with a marsupial, the quokka, Setonix brachyurus, in Western Australia. J. Hyg. (Lond), 71(3): 423-432.

35. Haley, B.J., Cole, D.J. and Lipp, E.K. (2009) Distribution, diversity, and seasonality of waterborne Salmonella in a rural watershed. Appl. Environ. Microbiol., 75(5): 1248-1255.

36. Parish, M.E. (1998) Coliforms, Escherichia coli and Salmonella serovars associated with a citrus-producing facility implicated in a Salmonellosis outbreak. $J$ Food Prot., 61(3): 280-284.

37. Moffartt, C.R., Lafferty, A.R., Khan, S., Kristeski, R., Valcanis, M., Powling, J. and Veitch, M. (2010) Salmonella Rubislaw gastroenteritis linked to a pet lizard. Med. J. Aust., 193(1): 54-55.

38. Jijon, S., Wetzel, A. and LeJeune, J. (2007) Salmonella enterica isolated from wildlife at two Ohio-rehabilitation center. J. Zoo. Wildl. Med., 38(3): 409-413. 